\title{
On the possibility of a shunt-stabilized superlattice terahertz emitter
}

\author{
Huidong Xu and Stephen W. Teitsworth ${ }^{\text {a) }}$ \\ Department of Physics, Duke University, Box 90305, Durham, North Carolina 27708-0305, USA
}

(Received 5 September 2009; accepted 18 December 2009; published online 11 January 2010)

\begin{abstract}
High field electronic transport through a strongly coupled superlattice (SL) with a shunting side layer is numerically studied using a drift-diffusion model that includes both vertical and lateral dynamics. The bias voltage corresponds to an average electric field in the negative differential conductivity region of the intrinsic current-field curve of the SL, a condition that generally implies space charge instability. Key structural parameters associated with both the shunt layer and SL are identified for which the shunt layer stabilizes a uniform electric field profile. These results support the possibility to realize a SL-based terahertz oscillator with a carefully designed structure. (C) 2010 American Institute of Physics. [doi:10.1063/1.3291614]
\end{abstract}

The gain for terahertz $(\mathrm{THz})$ radiation in semiconductor superlattices (SLs) related to Bloch oscillations has been theoretically studied since the 1970 s, ${ }^{1,2}$ suggesting that the SL can serve as an active medium for THz radiation. However, such a device has never been realized due to spacecharge instabilities associated with negative differential conductivity (NDC). This instability is manifested by moving field domains and self-sustained current oscillations with frequencies ranging up to hundreds of gigahertz, ${ }^{3}$ so the SL cannot be stably biased in the NDC region. Recently, SLbased $\mathrm{THz}$ oscillators have attracted renewed attention due to promising applications of $\mathrm{THz}$ technology and the need for high performance, compact, and room temperature $\mathrm{THz}$ sources. Current investigations of possible SL-based $\mathrm{THz}$ sources focus on a variety of schemes for stabilizing structures in the NDC region, e.g., using modulated bias ${ }^{4}$ or stacking SLs with small number of periods. ${ }^{5}$

The possibility of using a conductive shunt layer to stabilize a SL was recently suggested by Daniel $e t ~ a l .{ }^{6}$ Using a distributed nonlinear circuit model, they identified values of SL width and shunt resistivity for which static electric field domains are suppressed. However, this model is not capable of describing instability to moving field domains, ${ }^{3}$ since it does not include physical effects such as the dependence of tunneling currents on local charge density, or diffusive currents that flow parallel to the quantum wells of the SL. More recently, Feil et al. experimentally demonstrated the stabilization of a surface SL with effective width of approximately $10-20 \mathrm{~nm}$ using a lightly doped SL as shunt layer. ${ }^{7}$ Recently, we showed that a side shunting layer can stabilize a spatially uniform, time-independent electric field in weakly coupled SLs, provided that the lateral width of the SL-i.e., the spatial extent in the direction parallel to the quantum wells-is not too large. ${ }^{8}$ In this case, the transport occurs as sequential resonant tunneling of electrons between successive quantum wells. ${ }^{9}$ However, Bloch oscillations are expected for strongly-coupled SLs in which electronic transport occurs via miniband conduction. ${ }^{10,11}$ Here, we adapt the method for treating lateral dynamics in weakly coupled $\mathrm{SLs}^{8,12,13}$ to model transport in a strongly coupled SL with a shunting layer.

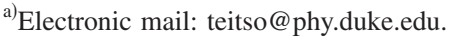

The structure is shown in Fig. 1(a). The SL is grown along the $z$ (vertical) direction and has lateral extent in the $x$ direction from zero to $L_{x}$, with a shunt of width $L_{\mathrm{s}}$ located at $x<0$. The extent in the $y$ direction is assumed large. The system evolves following the charge continuity equation:

$$
e \dot{n}(x, z, t)=-\nabla \cdot \mathbf{j}=-\frac{\partial j_{x}}{\partial x}-\frac{\partial j_{z}}{\partial z},
$$

and the Poisson equation

$$
\nabla \cdot \mathbf{F}=\frac{\partial F_{x}}{\partial x}+\frac{\partial F_{z}}{\partial z}=\frac{e}{\epsilon_{0} \epsilon_{\mathrm{r}}}\left(n-N_{\mathrm{D}}\right),
$$

where $e<0$ is the electron charge, $\epsilon_{0}\left(\epsilon_{\mathrm{r}}\right)$ is the free space (relative) permittivity, $n\left(N_{\mathrm{D}}\right)$ is the electron (ionized donor) density, $j_{z}$ is the vertical current density along the $z$ (growth) direction, $j_{x}$ is the lateral current density, and $\mathbf{F}$ denotes the electric field.

Using a well-established model, ${ }^{1,14,15}$ the vertical current inside the SL is

$$
j_{z}=e v\left(F_{z}\right) n-e D_{\|}\left(F_{z}\right) \frac{\partial[n]}{\partial z},
$$

where $v\left(F_{z}\right)$ is the local drift velocity which has Tsu-Esaki form, ${ }^{11}$
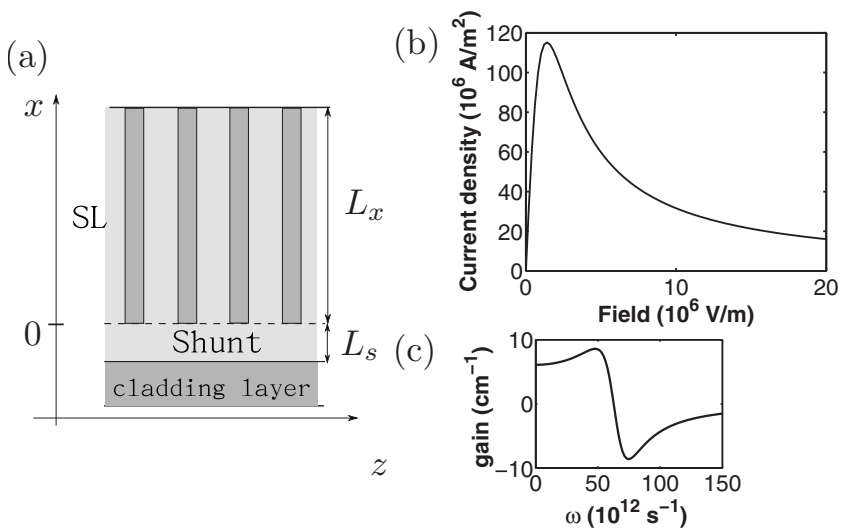

FIG. 1. (a) Schematic of the device structure: light shading denotes quantum wells and shunt; dark shading denotes barriers and a possible cladding layer. (b) The $J-F$ characteristic and (c) gain profile for the SL of structure 1, not including the shunt. 


$$
v\left(F_{z}\right)=\frac{2 v_{\mathrm{M}} F_{\mathrm{s}}}{1+F_{\mathrm{s}}^{2}} \quad F_{\mathrm{s}} \equiv F_{z} / F_{\mathrm{M}}
$$

and $D_{\|}$is field-dependent diffusion constant given by ${ }^{11}$

$$
D_{\|}\left(F_{z}\right)=\frac{k_{B} T}{e} \frac{v\left(F_{z}\right)}{F_{z}}=\frac{2 k_{B} T v_{\mathrm{M}}}{e F_{\mathrm{M}}\left(1+F_{\mathrm{s}}^{2}\right)} .
$$

Here, $F_{\mathrm{M}}$ denotes the field value for which the drift current exhibits a local maximum, cf. Fig. 1(b). The NDC in this current-field $(J-F)$ characteristic is associated with spatiotemporal instability, in which moving field domains lead to current self-oscillation. These phenomena have been wellstudied in Gunn diodes and unshunted SLs. ${ }^{10,14,15}$

At the emitter, the tunneling current is Ohmic with contact conductivity $\sigma, j_{z}(x, z=0, t)=\sigma F_{z}(x, z=0, t)$. The lateral current is also of drift-diffusion form;

$$
j_{x}=e \mu n F_{x}-e \frac{\partial\left(D_{\perp} n\right)}{\partial x},
$$

where $\mu$ denotes the low field electron mobility in the lateral direction. The shunt occupies one row of grid points in the simulation and is taken to have the same doping density as the SL. The charge continuity equation is similar but the drift part of the vertical current is linear in $F_{z}$ as follows:

$$
j_{z}^{(s)}=e \mu_{\mathrm{s}} n F_{z}-e \frac{\partial\left(D_{\mathrm{s}} n\right)}{\partial z},
$$

where $\mu_{\mathrm{s}}=\mu$ and $D_{\mathrm{s}}=D_{\perp}=D$ if the quantum wells and the shunt are made of the same material with bulk diffusion constant $D=k_{B} T \mu / e$. The boundary condition between shunt layer and SL is $j_{x}^{(s)}(z, t)=\lim _{x \rightarrow 0+} j_{x}(x, z, t)$, i.e., a high quality connection which could be realized, for example, using cleaved-edge overgrowth of the quantum well material. ${ }^{7,16,17}$

The system has multiple time scales. The dynamics in the shunt and in the lateral direction is $\epsilon_{0} \epsilon_{\mathrm{r}} /\left(e \mu N_{\mathrm{D}}\right) \approx 5$ fs for typical structures, much faster than the vertical dynamics in the SL (10 ps to $10 \mathrm{~ns})$. Therefore, an implicit method is used as in Ref. 8.

For the structures studied here, we set the critical electric field value to $F_{\mathrm{M}}=1.4 \times 10^{6} \mathrm{~V} / \mathrm{m}$, achievable at room temperature in InAs/AlSb SLs. ${ }^{5}$ The value of peak drift velocity $v_{\mathrm{M}}$ is variable and depends on well and barrier widths of the SL. ${ }^{14,18}$ We bias the structures in the NDC region, with an average field $U / l=6.67 \times 10^{6} \mathrm{~V} / \mathrm{m}$, where the total applied voltage $U=12 \mathrm{~V}$ is held constant and $l=1.8 \mu \mathrm{m}$ is the length of the SL in $z$ direction. We find that excess charge associated with accumulation or depletion layers in the SL moves laterally into the shunt mainly through diffusion, ${ }^{8}$ so the diffusion constant $D_{\perp}$ needs to be sufficiently high for the field to be stabilized. Since $D_{\perp}=k_{B} T / e \mu$, we can enhance the diffusion process by working at room temperature, $T$ $=300 \mathrm{~K}$. We assume a mobility $\mu=2 \mathrm{~m}^{2} / \mathrm{Vs}$, achievable using InAs quantum wells. ${ }^{17,19,20}$

The parameters for the structures studied are listed in Table I. There are two sets of $N_{\mathrm{D}}$ and $v_{\mathrm{M}}$, corresponding to different current density and absorption (gain). The gain is estimated with a semiclassical model at room temperature as follows 4,21
TABLE I. Parameters used for the shunted SL.

\begin{tabular}{llccc}
\hline \hline & $\begin{array}{c}L_{x} \\
(\mu \mathrm{m})\end{array}$ & $\begin{array}{c}N_{\mathrm{D}} \\
\left(10^{23} \mathrm{~m}^{-3}\right)\end{array}$ & $\begin{array}{c}v_{\mathrm{M}} \\
\left(10^{3} \mathrm{~m} / \mathrm{s}\right)\end{array}$ & $\begin{array}{c}\sigma \\
\left(e v_{\mathrm{M}} N_{\mathrm{D}} / F_{\mathrm{M}}\right)\end{array}$ \\
\hline Structure 1 & 7 & 0.8 & 9 & 1 \\
Structure 2 & 6 & 0.8 & 9 & 1 \\
Structure 3 & 5 & 0.8 & 9 & 1 \\
Structure 4 & 5 & 1.4 & 20 & 1 \\
Structure 5 & 2.5 & 1.4 & 20 & 1 \\
Structure 6 & 5 & 0.8 & 9 & 0.3 \\
\hline \hline
\end{tabular}

$$
g=-\frac{e d}{n_{\mathrm{r}} \epsilon_{0} c} \frac{j_{\mathrm{dc}}(e F d+\hbar \omega)-j_{\mathrm{dc}}(e F d-\hbar \omega)}{2 \hbar \omega} .
$$

The $J-F$ characteristic and gain for structures $1-3$ and 6 are shown in Figs. 1(b) and 1(c), respectively. The maximum value of the gain spectrum is about $9 \mathrm{~cm}^{-1}$ for these structures, and it is about $35 \mathrm{~cm}^{-1}$ for structures 4 and 5 .

The structure is biased with a uniform charge configuration $n=N_{\mathrm{D}}$ everywhere as initial condition. The left column of Fig. 2 shows snapshots of field and charge configuration at $x=L_{x}$ and the right column shows snapshots of the electron configuration $n$ for the whole structure after the system reaches static state or steady oscillation. The rows are for structure 1,2 , and 3, respectively, which have different $L_{x}$, but are otherwise identical. Structure 1 has a wide lateral size and demonstrates spatiotemporal instability. There is a static charge accumulation layer near the emitter. Closer to the collector, alternating charge accumulation and depletion layers form, move downstream, disappear at the collector and reappear inside the SL. For even wider SL, the oscillation is found to be chaotic (not shown). As the lateral size $L_{x}$ be-
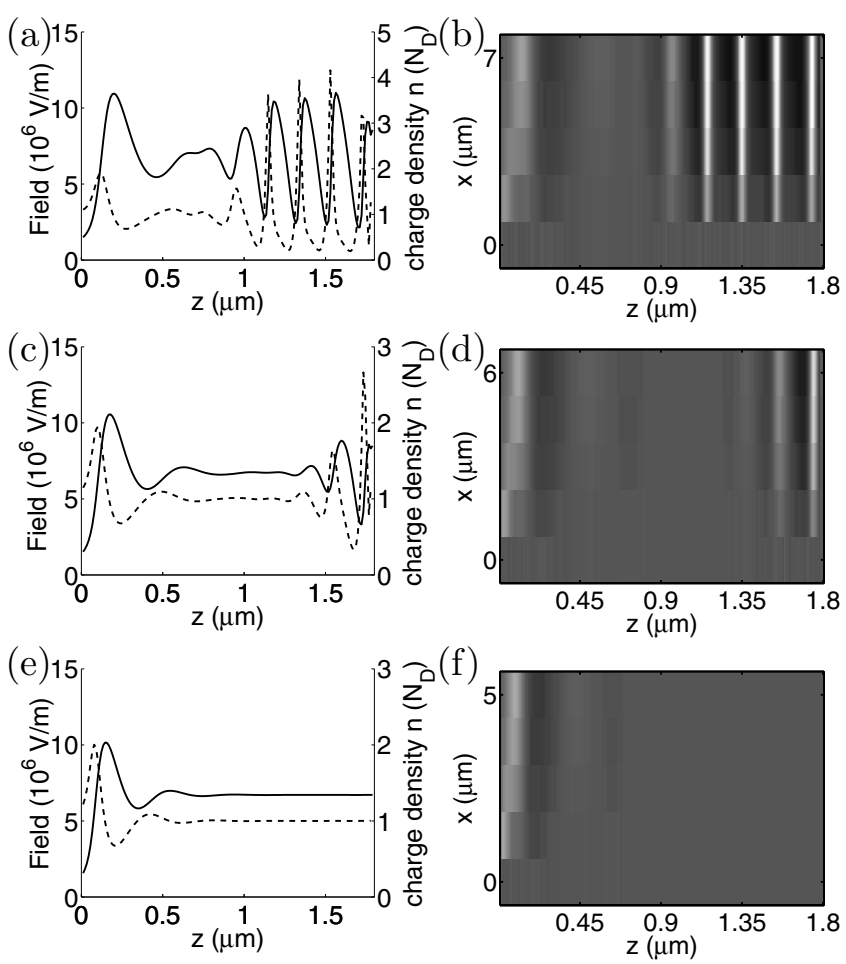

FIG. 2. Snapshots of field (solid line) and electron density (dashed line) configuration at $x=L_{x}$ (left column) and electron density vs $x$ and $z$ (right column) for structure $1[(\mathrm{a})$ and b)], structure $2[(\mathrm{c})$ and (d) $]$, and structure 3 $[(\mathrm{e})$ and (f)]. 
comes smaller, a region with relatively uniform field profile emerges between the emitter and collector regions, cf. structure 2 in Figs. 2(c) and 2(d). As the lateral size becomes even smaller, the field profile becomes static and nearly uniform, except near the emitter contact, as shown in Figs. 2(e) and 2(f).

The gain in Eq. (8) can be increased by increasing the doping density $N_{\mathrm{D}}$ or the peak drift velocity $v_{\mathrm{M}}$. However, this also increases the vertical current in the SL and the field configuration becomes more difficult to stabilize, i.e., $L_{x}$ needs to be even smaller. For example, structure 4, which has the same width as the stable structure 3 , is found to oscillate similar to structure 1 . For this combination of $N_{\mathrm{D}}$ and $v_{\mathrm{M}}$ one must reduce the lateral size to $L_{x} \lesssim 2.5 \mu \mathrm{m}$ (structure 5) in order to achieve shunt stabilization. We have also investigated the role of contact conductivity $\sigma$ on the shunt stabilization process. For structures 3 and 6 , identical except for their $\sigma$ values, we find that the field profiles and shunt stabilization are similar.

The shunt dissipates power without any optical gain, so to maximize the wall-plug efficiency, the current flowing in the shunt should be kept as low as possible. Since NDC in the total device current needs to be suppressed, a necessary condition is that the conductance of the shunt should be just high enough for this goal which provides an estimate of the threshold width of the shunt. For the shunt to work, the maximum magnitude of negative differential conductance of the SL should be smaller than the conductance in the shunt; $\mu N_{\mathrm{s}} L_{\mathrm{s}}>g_{\mathrm{m}} N_{\mathrm{D}} L_{x}$ or

$$
L_{\mathrm{s}}>g_{\mathrm{m}} L_{x} / \mu,
$$

where $g_{\mathrm{m}}=\max [|\partial v(F) / \partial F|]$ is the maximum absolute value of the NDC. Here, the doping density in the shunt $N_{\mathrm{s}}$ is taken equal to that in the SL $N_{\mathrm{D}}$. For structure $3, L_{\mathrm{s}}>4 \mathrm{~nm}$ using Eq. (9). Simulation shows that the threshold value of $L_{\mathrm{S}}$ is between 4 and $5 \mathrm{~nm}$. This width is even smaller than the period of the SL and quantum confinement effects may be significant.

In conclusion, we have numerically demonstrated that a strongly coupled SL can have uniform field biased in the NDC region stabilized by a shunting side layer, thus enabling a device that has positive gain in the $\mathrm{THz}$ regime. As the lateral size $L_{x}$, the peak velocity $v_{\mathrm{M}}$, or doping density $N_{\mathrm{D}}$ increases, the system becomes more difficult to stabilize and eventually loses stability to moving field domains. Electron diffusion in the lateral direction plays an essential role in the shunt stabilization process. In contrast, the contact conductivity does not have a strong effect. We have predicted shunt stabilization for structures with widths up to a few microns. In contrast, previous work showed that shunt stabilization can be effective in weakly coupled SLs with widths up to a few hundred microns, ${ }^{8}$ a difference largely due to the much smaller vertical current densities in those structures.

We gratefully acknowledge helpful conversations with Andreas Amann, Luis Bonilla, Jungsang Kim, and Eckehard Schöll. This work was supported by the National Science Foundation through Grant No. DMR-0804232.

${ }^{1}$ L. Esaki and R. Tsu, IBM J. Res. Dev. 14, 61 (1970).

${ }^{2}$ S. A. Ktitorov, G. S. Simin, and V. Y. Sindalovskii, Sov. Phys. Solid State 13, 1872 (1972).

${ }^{3}$ E. Schomburg, K. Hofbeck, J. Grenzer, T. Blomeier, A. A. Ignatov, K. F. Renk, D. G. Pavel'ev, Y. Koschurinov, V. Ustinov, A. Zhukov, S. Ivanov, and P. S. Kop'ev, Appl. Phys. Lett. 71, 401 (1997).

${ }^{4}$ T. Hyart, N. V. Alexeeva, J. Mattas, and K. N. Alekseev, Phys. Rev. Lett. 102, 140405 (2009).

${ }^{5}$ P. G. Savvidis, B. Kolasa, G. Lee, and S. J. Allen, Phys. Rev. Lett. 92 , 196802 (2004).

${ }^{6}$ E. K. Daniel, B. K. Gilbert, J. S. Scott, and S. J. Allen, IEEE Trans. Electron Devices 50, 2434 (2003).

${ }^{7}$ T. Feil, H.-P. Tranitz, M. Rienwald, and W. Wegscheider, Appl. Phys. Lett. 87, 212112 (2005).

${ }^{8}$ H. Xu, A. Amann, E. Schöll, and S. Teitsworth, Phys. Rev. B 79, 245318 (2009).

${ }^{9}$ H. Xu and S. Teitsworth, Phys. Rev. B 76, 235302 (2007).

${ }^{10}$ A. Wacker, Phys. Rep. 357, 1 (2002).

${ }^{11}$ L. L. Bonilla, R. Escobedo, and A. Perales, Phys. Rev. B 68, 241304(R) (2003).

${ }^{12}$ A. Amann and E. Schöll, Phys. Rev. B 72, 165319 (2005).

${ }^{13}$ A. Amann and E. Schöll, J. Stat. Phys. 119, 1069 (2005).

${ }^{14}$ E. Schomburg, T. Blomeier, K. Hofbeck, J. Grenzer, S. Brandl, I. Lingott, A. A. Ignatov, K. F. Renk, D. G. Pavel'ev, Yu. Koschurinov, B. Ya. Melzer, V. M. Ustinov, S. V. Ivanov, A. Zhukov, and P. S. Kop'ev, Phys. Rev. B 58, 4035 (1998).

${ }^{15}$ L. L. Bonilla and H. T. Grahn, Rep. Prog. Phys. 68, 577 (2005).

${ }^{16}$ W. Wegscheider, L. Pfeiffer, and K. West, Appl. Phys. Lett. 65, 2510 (1994).

${ }^{17}$ C. H. Möller, O. Kronenwerth, D. Grundler, W. Hansen, C. Heyn, and D. Heitmann, Appl. Phys. Lett. 80, 3988 (2002).

${ }^{18}$ A. A. Ignatov and Y. A. Romanov, Phys. Status Solidi B 73, 327 (1976)

${ }^{19}$ K. Ohtani and H. Ohno, Appl. Phys. Lett. 82, 1003 (2003).

${ }^{20}$ C. R. Bolognesi, H. Kroemer, and J. H. English, J. Vac. Sci. Technol. B 10, 877 (1992).

${ }^{21}$ A. A. Ignatov, E. Schomburg, J. Grenzer, S. Winnerl, and K. F. Renk, Superlattices Microstruct. 22, 15 (1997). 